\title{
PARTIAL VALIDATION OF CFD BLAST SIMULATION IN A CITYSCAPE ENVIRONMENT FEATURING STRUCTURAL FAILURE
}

\author{
STEPHANIE JEANNE BURROWS ${ }^{1}$, SHAUN ANTHONY FORTH ${ }^{1} \&$ ROBERT PHILIP SHELDON $^{2}$ \\ ${ }^{1}$ Cranfield University, Centre for Simulation and Analytics, UK. \\ ${ }^{2}$ Cranfield University, Centre for Defence Engineering, UK.
}

\begin{abstract}
We demonstrate the capabilities of computational fluid dynamics (CFD) and a pressure-impulse failure model to predict blast loading and structural damage in a geometrically complex cityscape. The simulated loading is compared against experimental results for $69 \mathrm{~g}$ PE4 in a $1 / 50^{\text {th }}$ scale model with wood-framed and plywood-faced buildings; data were collected from 11 pressure gauges throughout. In the initial simulation, geometric features were modeled as perfectly rigid, whereas buildings in the experiment failed: the resulting differences between the model and experiment allowed us to evaluate CFD when failure occurs. Simulated peak pressures during the first positive phase were still within $20 \%$ of experiment at most pressure gauges. However, errors in first phase impulses were around $40 \%$, suggesting that building-failure effects are greater toward the phase end. Then, to model building-failure effects, we attempted to fit pressure-impulse failure curves to the plywood-faces: this proved too simplistic to produce realistic blast wave behavior due to the various, complex failure modes. This work illustrates key limitations of available CFD software and the pressure-impulse failure model - both industry-standard tools to determine structural response to blast. We conclude that stronger coupling between blast loading and structural response is needed where significant failure occurs.
\end{abstract}

Keywords: CFD, finite-volume method, severe blast problems, structural failure, validation.

\section{INTRODUCTION}

The goal of a reinsurance-industry funded project has been to assess the financial impact of conventional explosive blasts in city-center locations [1]. Determining the financial loss associated with such events requires accurate estimates of the peak pressures and impulses on the surfaces of affected buildings; at Cranfield, our role has been to produce these values from the geometry of the environment and the properties and location of the explosive device.

Damage estimation tools currently used in the insurance industry tend to simplify blast physics. Common tools either ignore the effect of buildings or limit damage to structures with a direct line of sight to the charge location [2]. While useful for simple configurations, such approaches do not produce accurate loading predictions in built-up areas, where blast waves can be channeled along streets, reflected off buildings, and diffracted around corners [3, 4]. Such models may seek to address this by the empirical fix of expanding the area impacted by the blast to decrease the discrepancy between the model's damage estimates and reality.

In contrast, we use our blast modeling software, ProSAir [5], to predict how blast waves propagate through a cityscape by simulating the effects of the detonation of a high explosive charge, using a high-resolution, finite-volume scheme, as in the precursor Air3D code [6]. Good agreement has previously been demonstrated [7] between experiment [8, 9] and ProSAir's simulated results for a generic cityscape. 
The original aim of this work was to validate our simulation's pressure loading against experimental results from a scenario similar to those required for the reinsurance industry [1] - as we shall see, unexpected structural failures in the experiment forced us to look to incorporate such failure into our simulation.

In Section 2, we describe our reinsurance-relevant scenario and the experiment involving detonation of a charge and measuring blast loading in a $1 / 50^{\text {th }}$ scale model. Section 2.1 details how buildings close to the blast location failed. Section 3 outlines our initial computational analysis, in which we omit structural failure, and which includes quantification of discretization (numerical) errors and, necessarily limited comparison with experiment (including issues with experimental data). This shows that peak overpressure was well predicted but impulse was not. Then, in Section 4, we describe our attempt to fit a pressure-impulse failure model, originally designed for glazing elements, to the behavior of the plywood face panels in order to extend our validation to include the unexpected structural failure of the physical model. In Section 5, we summarize and conclude.

\section{EXPERIMENT}

A real-world scenario was selected that should demonstrate differences between the lineof-sight and more realistic models. It was simplified and anonymized by simplifying the building shapes to enable scale model construction and open publication - see Fig. 1 . A $1 / 50^{\text {th }}$ scale experimental model was built at the Cranfield Ordnance Test and Evaluation Centre (COTEC). Pressure gauges labeled with a P (shown in black in Fig. 1) had a direct line of sight to the charge, while those labeled with a $\mathrm{Q}$ (shown in magenta) did not.

As seen in Fig. 2, the model was framed using $47 \times 47 \mathrm{~mm}$ softwood, faced with $12 \mathrm{~mm}$ thick plywood using wood screws and secured to a raised plywood base to enable wiring of the pressure

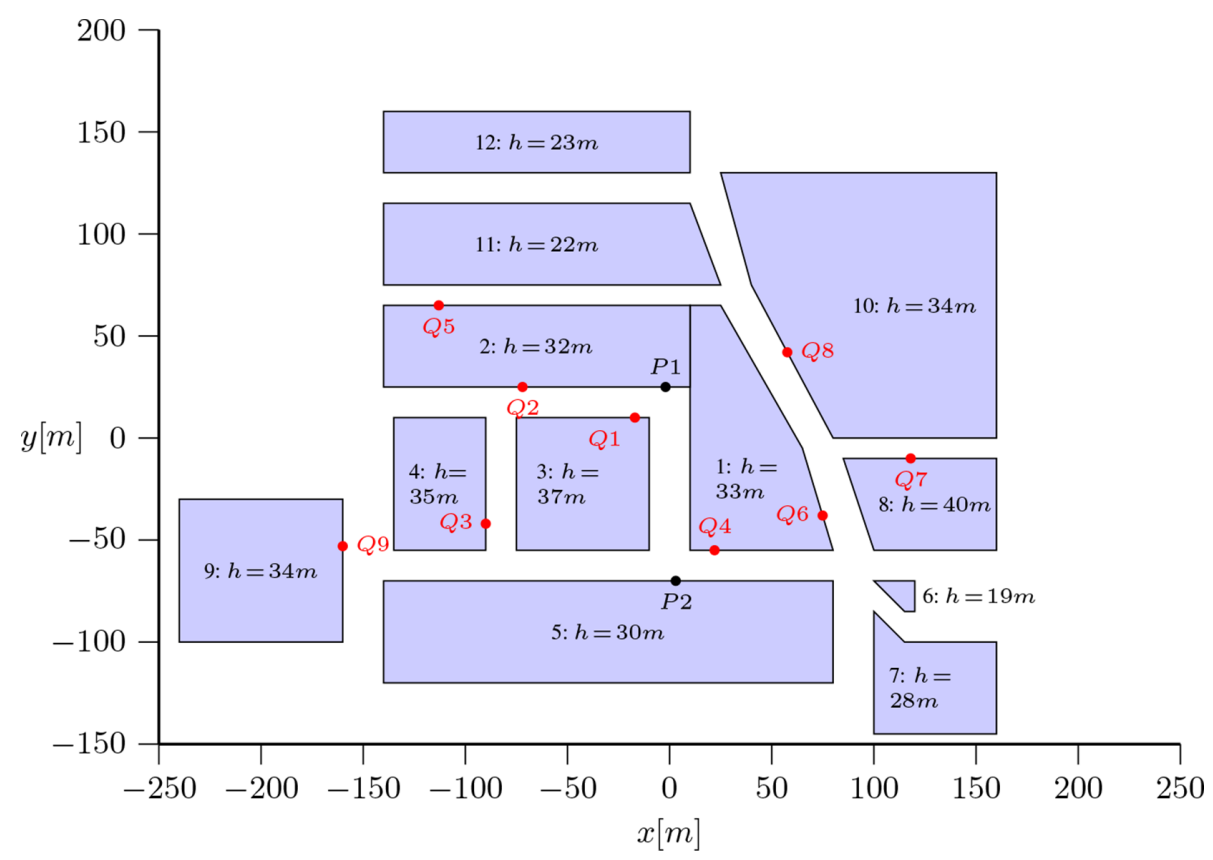

Figure 1: A diagram showing the building numbers, locations, heights $h$, and pressure-gauge locations of the full-size scenario. 


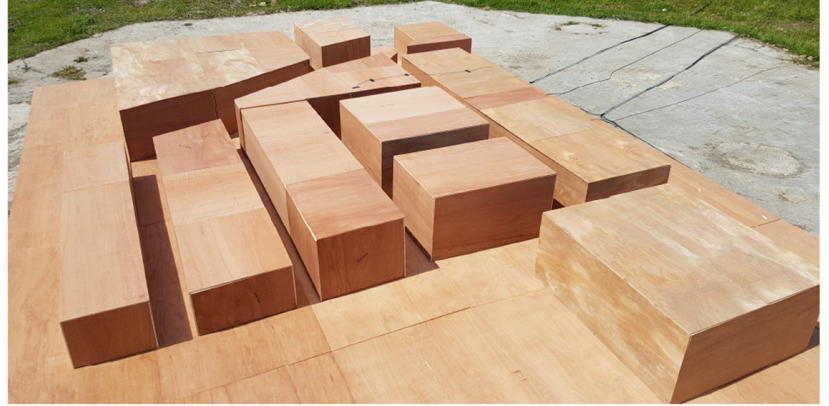

(a)

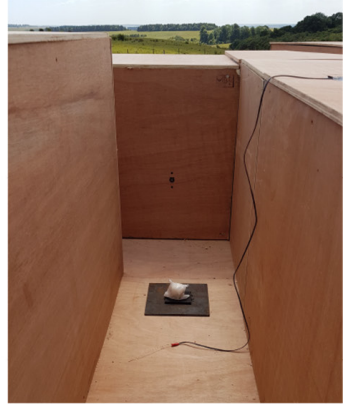

(b)

Figure 2: Photographs of the experimental setup before detonation. (a) $1 / 50^{\text {th }}$ scale model in plywood; (b) $69 \mathrm{~g}$ of PE4 on $20 \mathrm{~mm}$ polystyrene and $4 \mathrm{~mm}$ mild steel. The hole for a pressure gauge can be seen on the wall behind the charge.

gauges. The charge was $69 \mathrm{~g}$ of PE4, detonated by an L2A2 detonator giving a total TNT-equivalent mass of $89.6 \mathrm{~g}$ (equating to a full-scale $11200 \mathrm{~kg}$ TNT charge). The charge was placed on a $4 \times 200 \times 200 \mathrm{~mm}$ mild steel plate to minimize damage to the plywood base. The charge was raised $20 \mathrm{~mm}$ off the plate using a piece of polystyrene so that the charge's center was at a height of $40 \mathrm{~mm}$, corresponding to the $2 \mathrm{~m}$ of the full-size scenario.

\subsection{Experimental results}

During detonation, overpressure data were collected at the 11 pressure gauges (see [10]), at the locations of Fig. 1. These data are presented alongside the simulation results in Section 3.

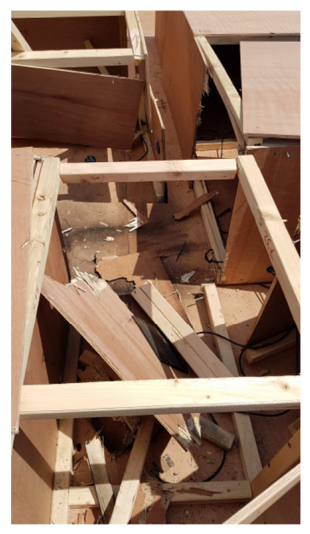

(a)

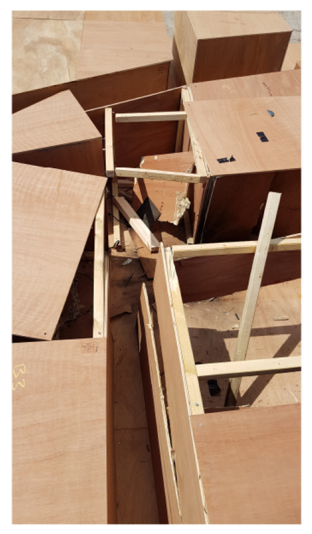

(b)

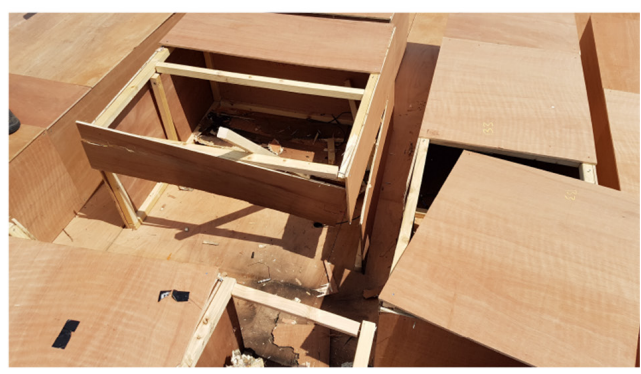

(c)

Figure 3: Photographs showing the area damaged by the charge from different viewpoints: (a) from the alley between buildings 1 and 10, looking in the negative $x$-direction; (b) from the alley between buildings 2 and 4, looking in the positive $x$-direction; and (c) from the corner of buildings 1, 10, and 11, looking toward the bottom-left corner of Fig. 1. Some buildings moved away from their initial position and some had roof panels removed by hand to retrieve pressure gauges. 
Figure 3 shows damage that resulted from the blast. The damage was so significant as to prevent repeat experiments. Building 3 in Fig. 1 detached from the model base and slid toward building 4. Some frame members failed in bending (e.g. the blast-adjacent face of building 5 buckled, with the kink oriented along the vertical axis through P2). Other members failed in shear. Fixing failures occurred as the screws were ripped out. Failure was an unintended result, complicating model validation, as highlighted in Sections 3 and 4.

\section{ANALYSIS AND COMPARISON}

As a comparison, simulations of the full-scale scenario were run at different grid resolutions, using a $11200 \mathrm{~kg}$ TNT charge. The solid steel plate situated beneath the blast in the experiment was included (though it was not resolved at coarser grid resolutions).

The grid resolutions used were $2.00 \mathrm{~m}, 1.00 \mathrm{~m}, 0.50 \mathrm{~m}$, and $0.25 \mathrm{~m}$. All were run using a $400 \times 400 \times 100 \mathrm{~m}$ domain except for the finest grid resolution, for which a $200 \times 200 \times 100 \mathrm{~m}$ domain was used due to desktop PC memory limitations. This means that some pressure gauges were excluded from the finest grid resolution simulation.

Following convention, we define phase 1 as the period after the arrival of the blast wave and before the overpressure becomes negative. Phase 2 is the period after the overpressure becomes negative and before it becomes positive again. Typically, phase 1 is most important for assessing a building's structural response. However, the effects of phase 2 and later phases may be significant, especially when assessing complex citycenter locations, where channeling between, and reflections off, buildings can increase their magnitude $[3,4]$.

Key blast parameters are a phase's arrival time, peak pressures, and impulse. Because of noise in the experimental overpressure measurements, identifying the start of a new phase at each sign change would result in many, short phases. This would hinder comparison between experimental and simulated results: corresponding overpressure time series might be in reasonable agreement, but phase blast parameters would show large error. To overcome this, we first set a phase change tolerance of $1 \%$ of the maximum absolute value of the impulse observed before some cut-off time. The cut-off time was set to the simulated time of arrival for phase 4 to prevent choosing an exceptionally high maximum impulse based on damaged pressure gauges. As impulse is the integral of overpressure, its extrema corresponds to zeros of overpressure; we identify phase arrival times as the peaks in the impulse with prominence greater than the phase change tolerance. This is described in detail in [10].

In Sections 3.1 and 3.2, simulations using a succession of finer meshes were used to estimate their mesh convergence and discretization error with respect to an idealized physical model. This assesses the impact of both space and time discretization, as the timestep is proportional to the cell size divided by the local physical wave speed by the well-known CFL condition [11]. The error between the experimental and simulation results is a combination of this discretization error and the modeling error between the idealized model and reality. Where the modeling error dominates, the reasons are discussed in Sections 3.3 and 3.4.

The remainder of this section focuses on phase 1, as the experimental and simulation overpressure records, and so blast parameters, diverged thereafter as blast reflections were affected by building movement and failure. These effects increase with time, and the simulated model was no longer appropriate for comparison after phase 1. 


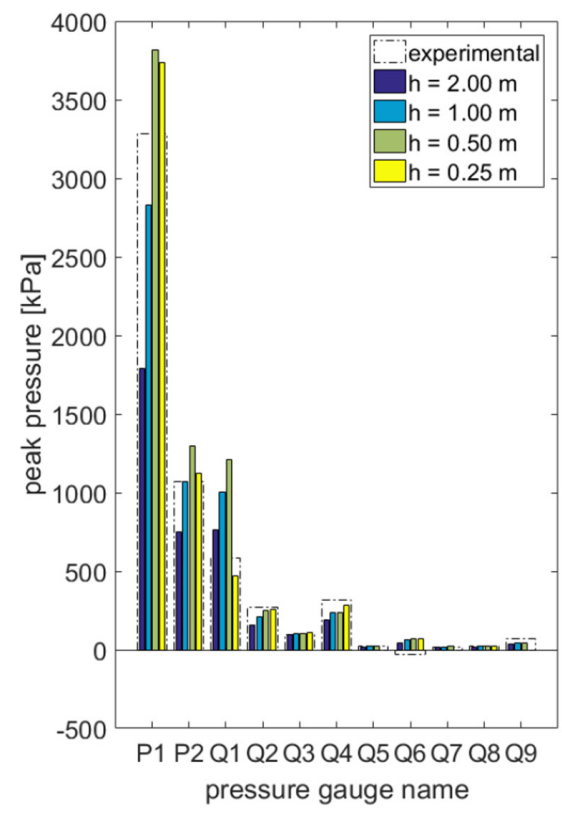

(a)

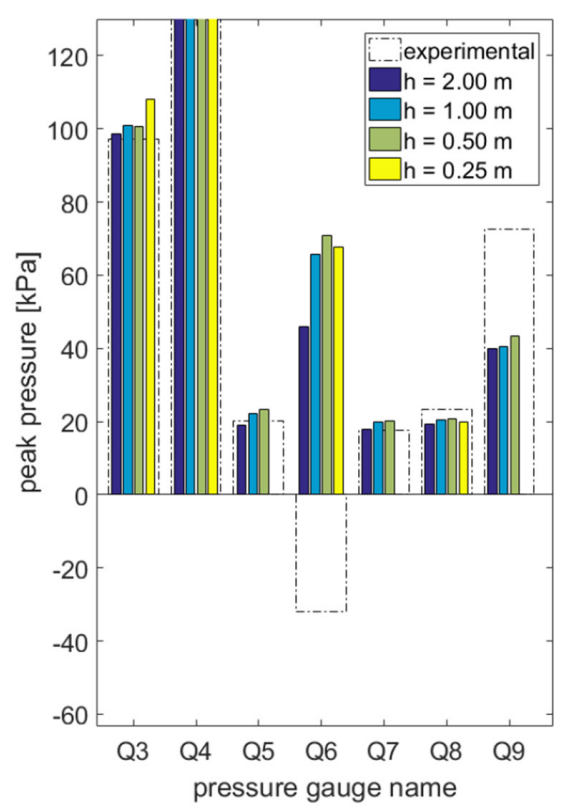

(b)

Figure 4: (a) The peak pressure during phase 1 for each pressure gauge at varying grid resolutions; (b) An enlarged plot of pressure gauges Q3-Q9; the peak pressure for Q4 exceeds the bounds of this plot.

\subsection{Peak pressure during phase 1}

Figure 4a shows the simulated, phase 1, gauge peak pressures for various grid resolutions together with the experimental results; a close-up on the peak pressures at Q3 and Q5-Q9 is in Fig. 4b (data for Q4 are truncated).

While none of the Q pressure gauges had a line of sight to the explosive charge, both simulation and experiment show significant overpressures. While the P pressure gauges generally experienced higher overpressures, assuming no loading at locations without line of sight to the blast would produce inaccurate predictions.

Apart from Q1, Q6, and Q9, each gauge showed reasonable agreement between simulations at varying grid resolutions, and between simulation and experiment. The significant drop in Q1's overpressure from $h=0.50 \mathrm{~m}$ to $h=0.25 \mathrm{~m}$, and Q6's unexpectedly negative experimental value are considered in Sections 3.3 and 3.4. Q9 shows good numerical convergence but significant modeling error when compared to experiment. However, this point was not contained within the finest grid-resolution simulation and so might not be well converged (consider Q1 were the $h=0.25 \mathrm{~m}$ simulation to be absent).

Given peak pressures, $P_{h}$, calculated on meshes with cell sizes, $h=2.0 \mathrm{~m}, 1.0 \mathrm{~m}, 0.5 \mathrm{~m}$, and $0.25 \mathrm{~m}$, we estimate the relative discretization error to be $\left|P_{h}-P_{h / 2}\right| /\left|P_{h / 2}\right|$. Figure 5a shows these estimated discretization errors were below $20 \%$ at the finest grid resolution apart from those points already identified. Differences between simulation and experiment in excess of $20 \%$ should be due to modeling error. 


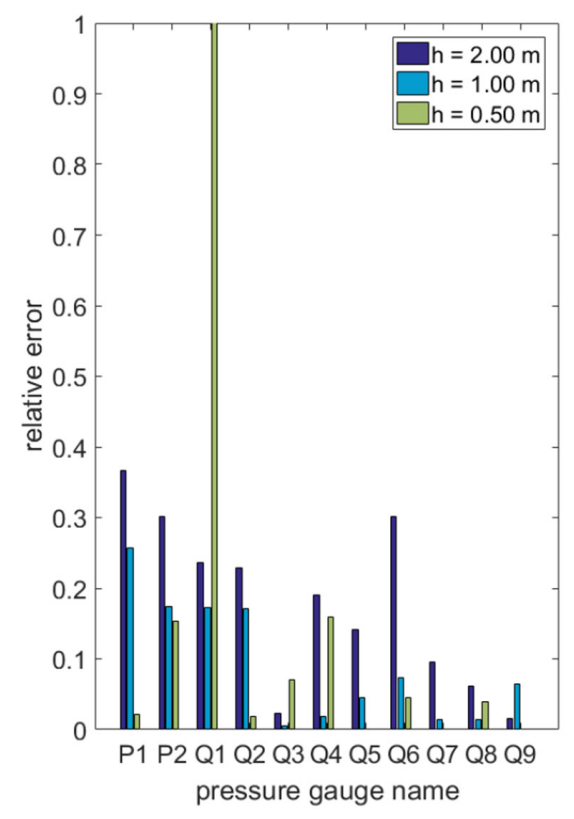

(a)

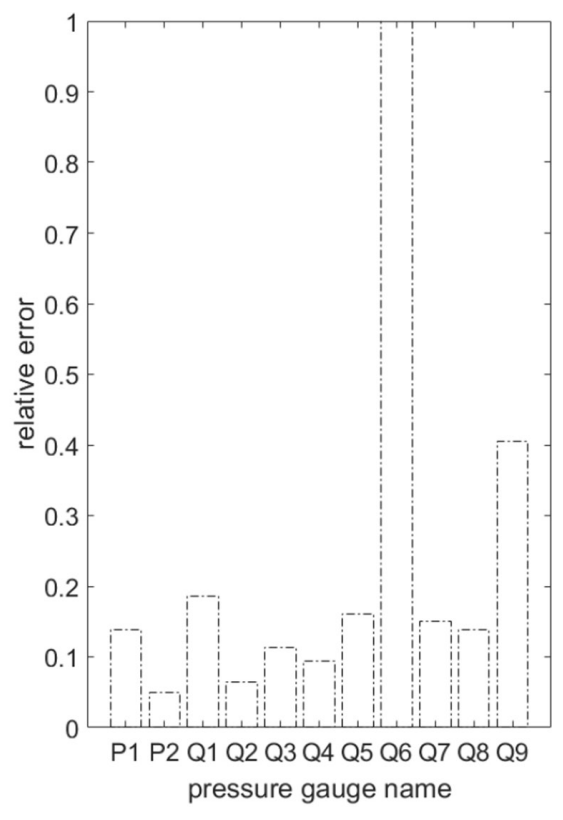

(b)

Figure 5: (a) Relative discretization error in phase 1 peak pressure for various grid resolutions; (b) Relative error at finest grid resolution with respect to the experimental value. The vertical bounds in each plot are limited to 1.0.

The relative errors in the finest available grid resolution with respect to the experimental values are shown in Fig. 5b; Q5, Q7, and Q9 were all outside the domain of the $h=0.25 \mathrm{~m}$ simulation, so the $h=0.50 \mathrm{~m}$ values were used. All of the errors were below $20 \%$ apart from for Q6 and Q9. At all other locations, the relative error between the simulated and experimental peak pressures was below the estimated discretization error, this indicates that essentially all of the error in the phase 1 peak pressure is accounted for by discretization error. In other words, there is good agreement between the idealized model and the experiment, at least up until the phase 1 peak pressure is reached.

\subsection{Impulse during phase 1}

Figure 6 shows the phase 1 impulse at varying grid resolutions for each pressure gauge along with the experimental impulse. The estimated relative discretization errors are shown in Fig. 7a. The error between the experimental and simulated impulse on the finest grid is in Fig. 7b. The experimental impulses were scaled up to the full-sized scenario.

The phase 1 impulse is the integral of the overpressure over the phase. We expect smaller discretization errors in the phase impulse than in the peak pressure because integration smoothes out fine-scale differences in the overpressure time series. This causes the estimated relative discretization error for impulse $(\approx 10 \%)$ to be much lower than for peak pressure $(\approx 20 \%)$ at most pressure gauges. However, the errors between the simulated and experimental phase 1 impulses were around 40\%; because these are larger than the estimated discretization errors, this indicates that modeling errors dominate for the impulse. 


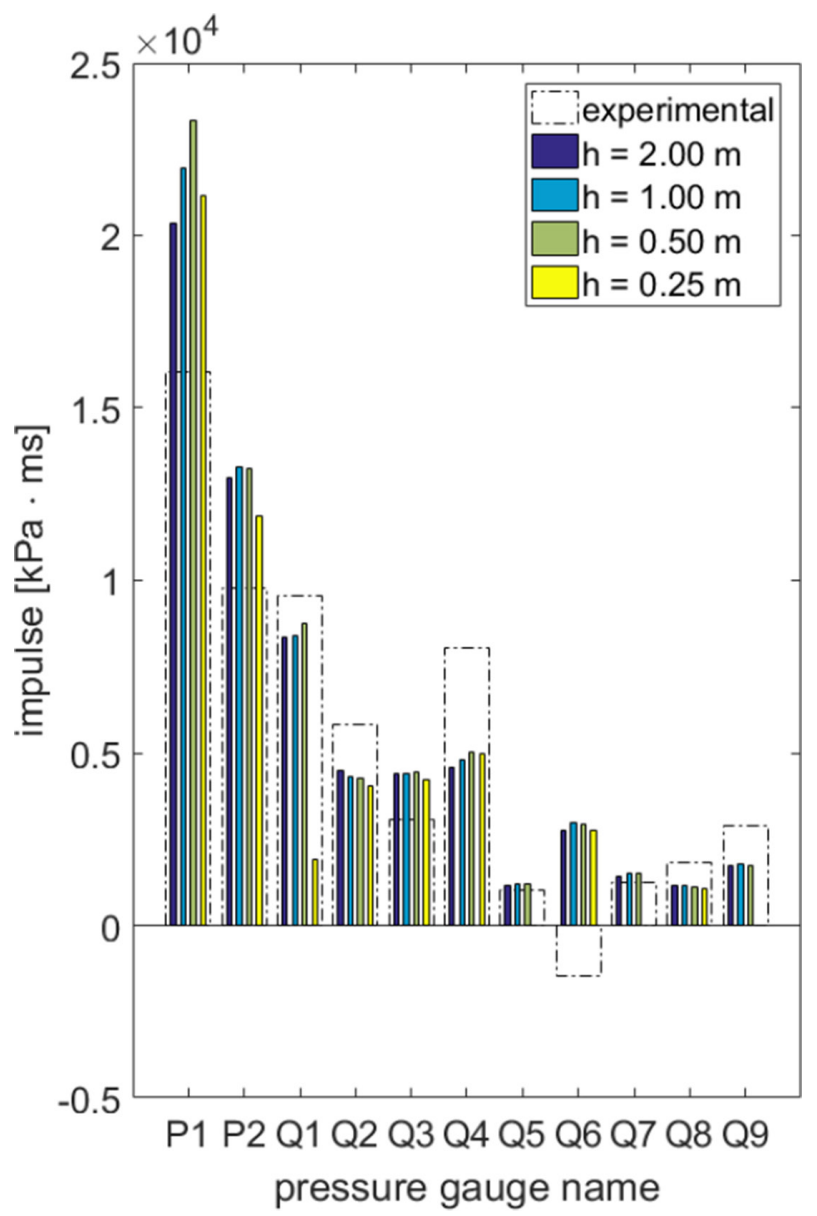

Figure 6: Impulse for phase 1 for each pressure gauge at varying grid resolutions. The experimental impulse is also shown.

An important difference between model and experiment was the experimental building failure and movement. The peak pressure appears largely unaffected by this difference but the impulse, taken over the full first phase, is.

In Sections 3.3-3.4, we examine the reasons for the high estimated discretization error in both overpressure and impulse for gauge Q1 and the high modeling error for gauge Q6.

\subsection{Errors due to changing phase duration at gauge Q1}

Figure 4 shows a large drop in the phase 1 peak pressure for Q1 between the $h=0.50 \mathrm{~m}$ and $h=0.25 \mathrm{~m}$ grid resolutions. There was also poor agreement between the simulated and experimental peak pressures. To understand the underlying cause, consider Fig. 8, which shows the simulated and experimental overpressure time series.

There are two issues evident in Fig. 8. First, the finest grid resolution had an extra, very brief, negative phase, starting at about $29 \mathrm{~ms}$, compared to the other simulations and experiment. The coarser mesh simulations show the first positive phase starting at about $11 \mathrm{~ms}$, 


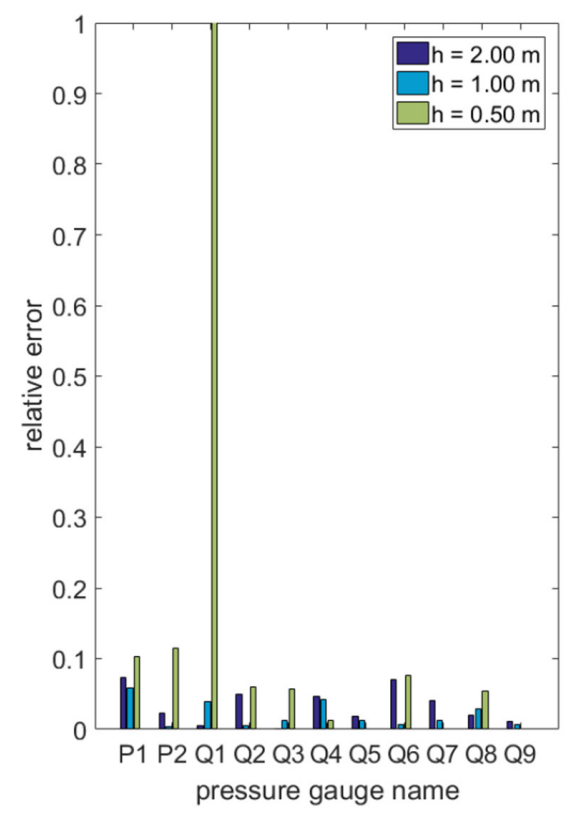

(a)

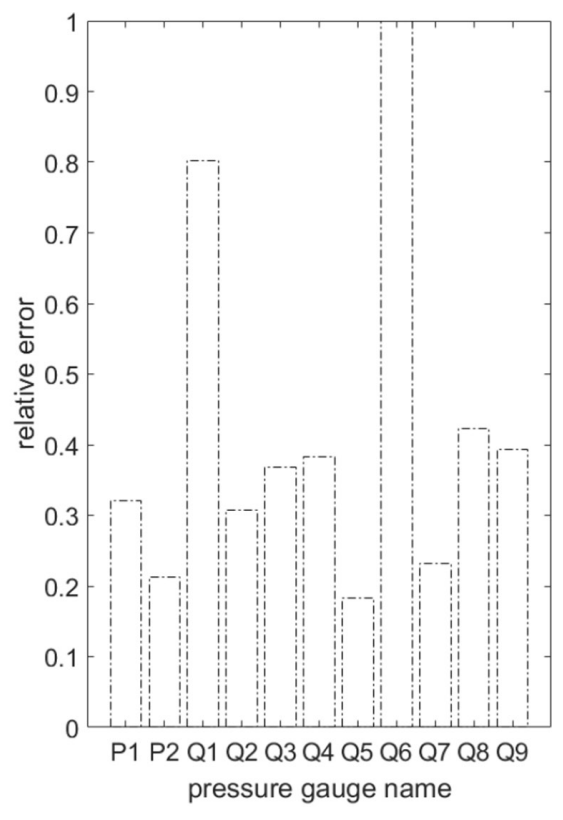

(b)

Figure 7: (a) Convergence of estimated relative discretization error in impulse over successive grid resolutions; (b) Relative error in finest grid resolution with respect to the experimental value.

a positive local minimum at around $29 \mathrm{~ms}$ and the phase ending at around $55 \mathrm{~ms}$. The second peak in overpressure at $31 \mathrm{~ms}$ of about $800-1200 \mathrm{kPa}$ is the maximum overpressure for these simulations. However, on the finest mesh, there is a brief period of underpressure at $29 \mathrm{~ms}$ : this divides the coarser meshes' long positive phase into two shorter positive phases, separated by a momentary negative phase at $29 \mathrm{~ms}$. The maximum overpressure for phase 1 is about $500 \mathrm{kPa}$ at $13 \mathrm{~ms}$, while the second positive phase has a peak of $1100 \mathrm{kPa}$

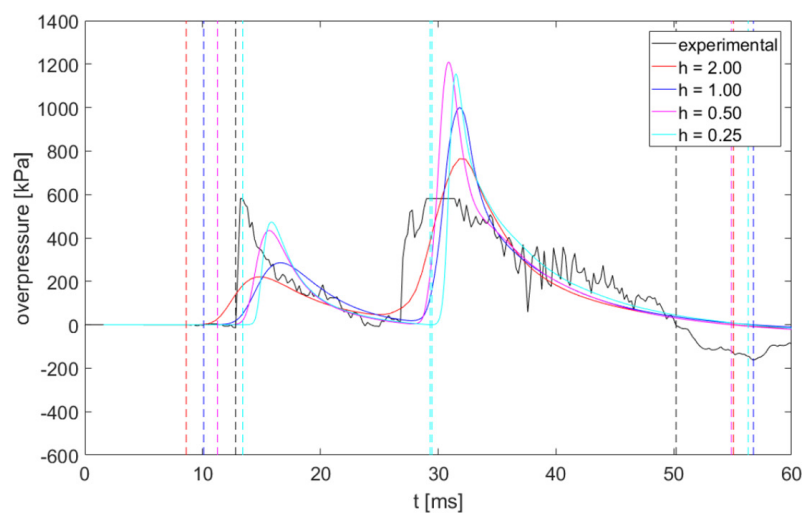

Figure 8: Time series of the overpressure at Q1. The time axis has been scaled so that values are those associated with the full-scale scenario. The time of arrival of each phase for each data set is shown as a dashed vertical line. 


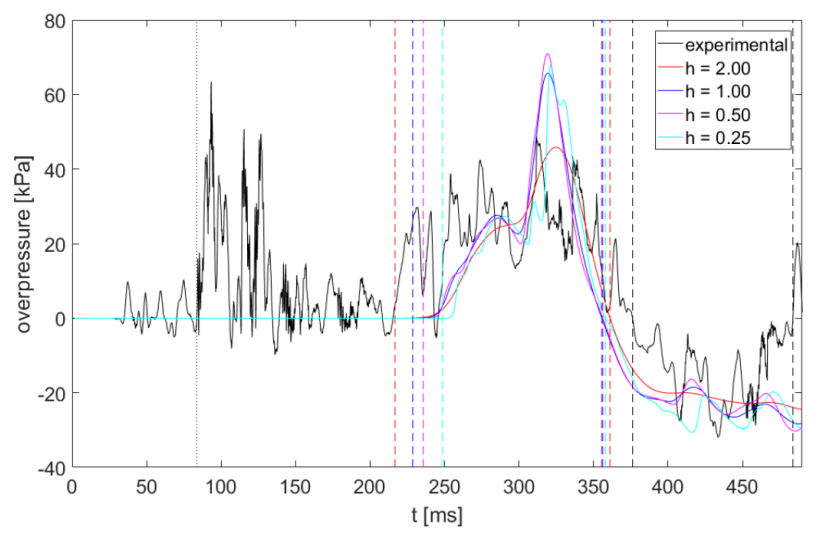

Figure 9: Time series of the overpressure at Q6.

at $31 \mathrm{~ms}$. We see that the phase 1 peak pressure is associated with the second peak in phase 1 overpressure on the coarser meshes but with the first at the finest grid resolution. If the overpressures at the second peak at the two finest grid resolutions are compared, the error is around $5-10 \%$.

This illuminates a point: the maximum peak pressure does not necessarily occur during the first positive phase. In complex geometries, diffracted and reflected waves can interact to produce unusual blast-wave shapes. This means that it is likely necessary to look past the first positive and negative phases to predict building damage.

The second issue with Q1 was that the experimental pressure gauge artificially saturated at around $600 \mathrm{kPa}$, as shown by the flat line at around $30 \mathrm{~ms}$ in Fig. 8. This caused the large differences between the experimental and simulation results.

\subsection{Errors due to noisy experimental data}

In Figs. 6 and 7b, Q6 also showed a large difference between the experimental and simulated phase 1 peak pressures. Looking at the time series of Fig. 9, we see that there is a poor correspondence between the simulated and experimental data: apparent times of arrival differ from $25 \mathrm{~ms}$ in the experiment to $200-250 \mathrm{~ms}$ in the simulation. Our automated post-processing of the experimental data estimates a phase time of arrival out of step with those of the simulation. The experimental time of arrival at around $80 \mathrm{~ms}$ is some $140 \mathrm{~ms}$ earlier than that of the simulation. For this to occur, the blast wave would need to travel around $137 \mathrm{~m}$ over the top of building 1 from the initial blast location to Q6. Using the UN Safeguard Kingery-Bulmash Blast Parameter Calculator [12], the arrival time at this distance for a $11200 \mathrm{~kg}$ TNT blast over level ground is around $250 \mathrm{~ms}$. This is in line with the simulation results and shows that the experimental overpressures from 80 to $220 \mathrm{~ms}$ result from some undesired effect in the experiment.

If we apply the arrival times from the simulation to the experiment, then the peak pressure for phase 1 is $48.67 \mathrm{kPa}$ with an error of $39.4 \%$ compared to the simulation and the impulse is $2884.7 \mathrm{kPa} \cdot \mathrm{ms}$ with an error of $4.91 \%$. To explain the still large error in peak pressure, consider that Q6 was on the far side of building 1, which was immediately adjacent to the blast. As seen in Fig. 3, the buildings adjacent to the blast were significantly damaged, with faces and tops ripped off, and buildings sliding away from their initial positions. We believe that the early overpressure measurements for the experimental time series at Q6 were associated with vibrations traveling through either the building or the plywood base of the experimental 
model. It is also possible to observe these fast vibrations arriving ahead of the simulated blast wave for other pressure gauges situated on the far sides of buildings adjacent to the blast (for brevity, graphs not shown).

\section{INCORPORATING FAILURE}

One explanation for the differences in the phase 1 impulse (and all bulk quantities for later phases) between the simulated and experimental results is that failure affects the overpressure that reaches the pressure gauges. We aimed to use ProSAir's simple pressure-impulse curve failure model to investigate this effect.

It was first necessary to determine the required failure model such that the correct plywood panels failed. Since failure was unintended, this was done retrospectively; the process is described in the following sections.

\subsection{ProSAir's failure model}

Failure was incorporated using a single pressure-impulse iso-damage curve [13] as a failure model as in Air3D [6] for failure of glazed elements (called windows here). Such elements start as a single layer of solid cells in the computational mesh. The maximum spatially averaged pressure loading and impulse during each phase is tracked; when these exceed the threshold of a user-specified P-I relationship, the window element is removed from the simulation.

We make use of the PI-Asymptotes model, described in the ProSAir User Guide [7]. The user specifies the pressure $\left(p_{\text {asymp }}\right)$ and impulse asymptotes $\left(I_{\text {asymp }}\right)$ of the P-I failure curve; if both the peak phase pressure and phase impulse exceed their corresponding asymptotes, the window fails, otherwise not. Choosing an appropriate model for our experiment requires the choice of $p_{\text {asymp }}$ and $I_{\text {asymp }}$ such that the correct panels fail.

\subsection{Specification of panels subject to failure}

Although the observed modes of failure for the buildings were varied and complex, we sought to simplify this by categorizing the failure into two types: wall failure or roof failure. Wall failure involves buckling or shearing of the facing panels and often failure of the supporting joists. Roof failure occurs after a wall has failed, when blast ingress blows the roof off of the building (i.e. securing screws fail). A failure model was used to describe the wall-failure criteria. For the roof, it was assumed that failure depends on the force across a panel (rather than pressure), as failure occurs at screws at the corners, rather than in the body of the panel.

Wall and roof panel placement were reconstructed from photographs, based on construction using discrete plywood panels. The simulated wall and roof positions are shown in Fig. 10. The panel thickness was $12 \mathrm{~mm}$. To avoid gaps between panels, each panel is surrounded by a $12 \mathrm{~mm}$ wide rim of solid cells as indicated in Fig. 10, where the size of each panel is shown.

Roof failure depends on wall failure - the building tops cannot be blown off if there is no blast ingress from failing wall panels. Because of this, we first determine appropriate criteria for wall failure, with roof response absent. We then shift our focus to roof failure.

\subsection{Determining failure criteria for walls}

We started by running a simulation to determine the maximum average pressure and impulse reached on each wall panel without failure. The simulation used a grid cell size of $1.0 \mathrm{~cm}$ and a domain such that $-3.0 \mathrm{~m} \leq x \leq 3.0 \mathrm{~m},-3.0 \mathrm{~m} \leq y \leq 3.0 \mathrm{~m}$ and $0.0 \mathrm{~m} \leq z \leq 2.0 \mathrm{~m}$. 


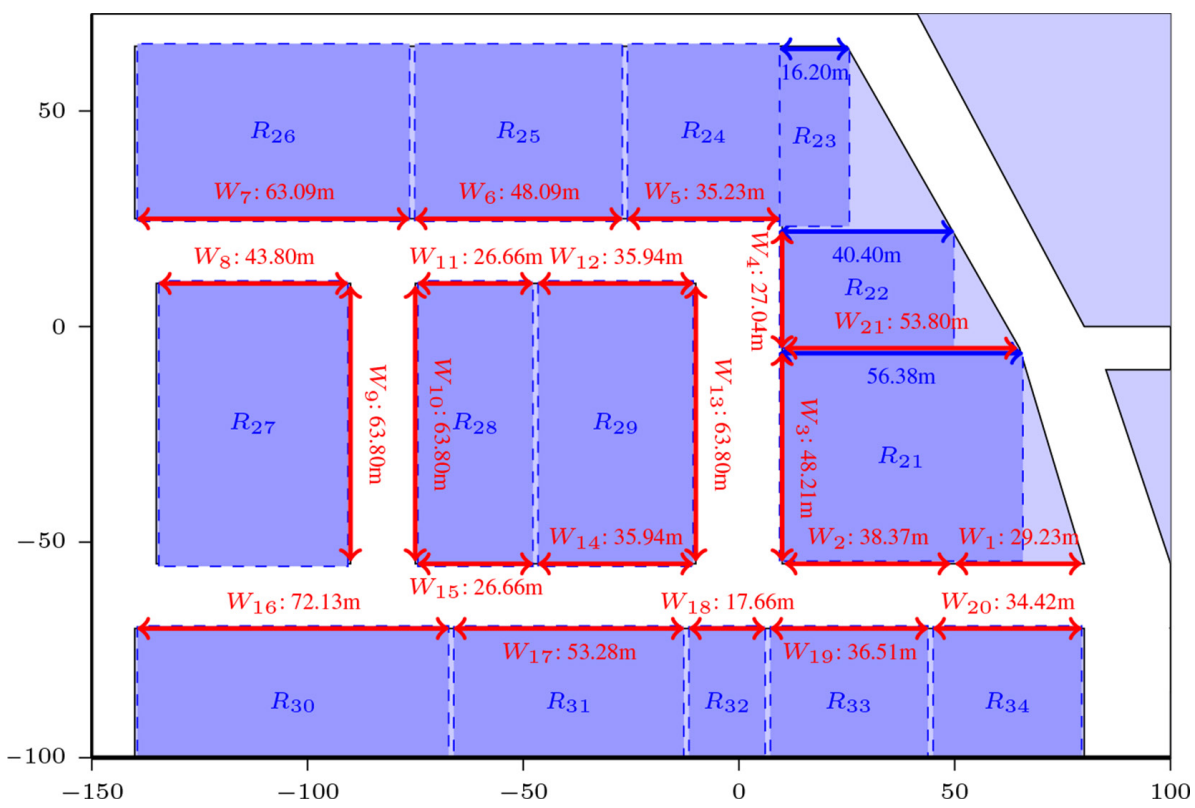

Figure 10: Position and labeling of the plywood wall panels, labeled $W_{1}$ to $W_{20}$, and roof panels, labeled $R_{21}$ to $R_{34}$, subject to the failure models in the simulations incorporating failure.

Thick solid lines in Fig. 11 show phase peak pressure as a function of phase impulse for each wall panel; as time passes, each panel moves along its curve from the bottom left toward the top right, reaching the maximum pressure versus maximum impulse point at the end of the phase. Major failure occurred on panels 4, 5, and 13, so the failure criteria should be asymptotes that separate the ends of these trajectories from those of the non-failing panels; the relevant thresholds are shown as dashed lines in Fig. 11.

There are different choices of $\left(I_{\text {asymp }}, p_{\text {asymp }}\right)$ that cause the correct failure behavior of panels. For the correct panels to fail, both $p_{\text {asymp }} \leq 1019.81 \mathrm{kPa}$ and $I_{\text {asymp }} \leq 247.88 \mathrm{kPa} \cdot \mathrm{ms}$ are needed. For correct survival, we need either $p_{\text {asymp }} \geq 560.82 \mathrm{kPa}$ or $I_{\text {asymp }} \geq 227.85 \mathrm{kPa} \cdot \mathrm{ms}$. The gray region of Fig. 11, which is very narrow for peak pressures less than $560.82 \mathrm{kPa}$, is where $\left(I_{\text {asymp }}, p_{\text {asymp }}\right)$ satisfy both these criteria. We could explore different values of both $p_{\text {asymp }}$ and $I_{\text {asymp }}$; however, if $I_{\text {asymp }} \geq 227.85 \mathrm{kPa} \cdot \mathrm{ms}$ (required for a low value of $\left.p_{\text {asymp }}\right)$, then the failing panels will pass the pressure threshold before passing the impulse threshold as they travel along their trajectories from bottom left to top right. There is no practical difference between $p_{\text {asymp }} \geq 560.82 \mathrm{kPa}$ and $p_{\text {asymp }} \leq 560.82 \mathrm{kPa}$ : failure occurs when the impulse threshold is reached, regardless. In the following, we choose $p_{\text {asymp }}>560.82 \mathrm{kPa}$ and vary $I_{\text {asymp }}$.

We will consider the three choices of failure criteria of Table 1.

- For the high impulse failure criteria, $I_{\text {asymp }}$ is chosen to separate the failed wall panel $W_{13}$ from the survived $W_{18}$ and a low value of $p_{\text {asymp }}$ is used; panels will fail late in the first positive phase and after peak pressure.

- For the low impulse case, the impulse asymptote is reached almost instantly and failure occurs when the pressure asymptote is reached; panels will fail almost as soon as the blast wave impacts. 


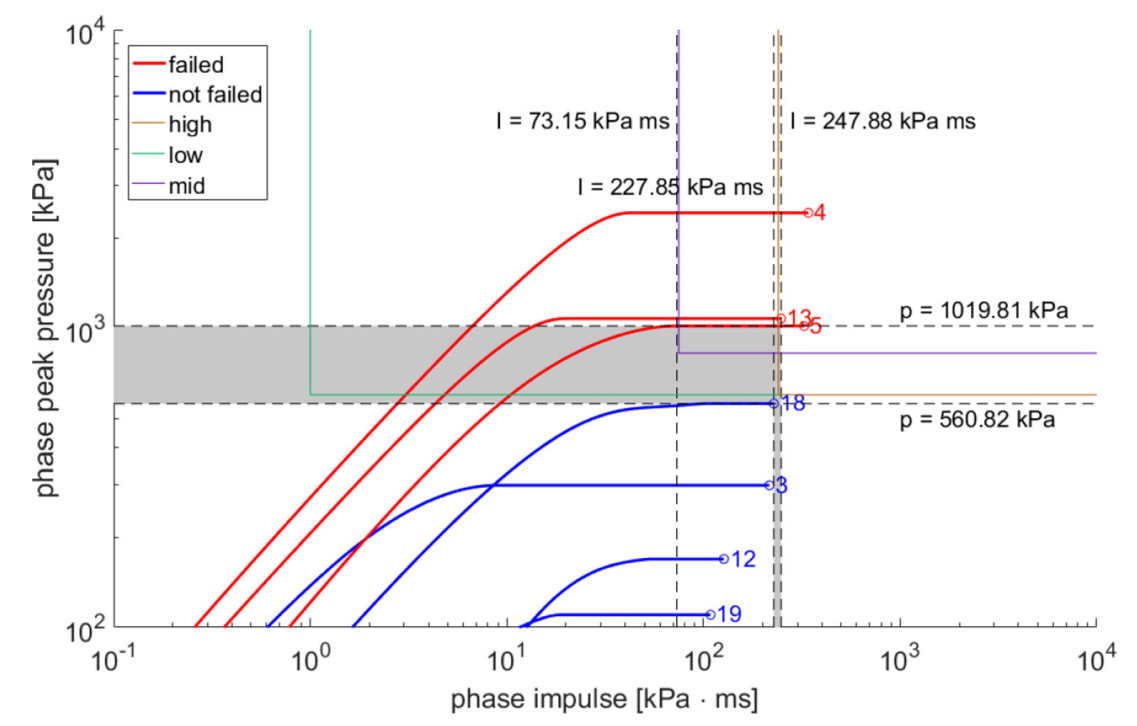

Figure 11: The maximum spatially averaged pressure and impulse reached on each plywood wall panel, labeled as in Fig. 10, during a $1.0 \mathrm{~cm}$ cell size simulation with no failure, showing the limiting values for the pressure and impulse asymptotes for wall failure. The failed panels 4, 5, and 13 may be separated from the others using pressure and impulse asymptotes provided $p_{\text {asymp }} \leq 1019.81 \mathrm{kPa}$ and $I_{\text {asymp }} \leq$ $247.88 \mathrm{kPa} \cdot \mathrm{ms}$, and either $p_{\text {asymp }} \geq 560.82 \mathrm{kPa}$ or $I_{\text {asymp }} \geq 227.85 \mathrm{kPa} \cdot \mathrm{ms}$ (i.e. the point $\left(I_{\text {asymp }}, p_{\text {asymp }}\right)$ must be within the gray region). The three failure criteria of Table 1 are shown for reference.

- For the mid impulse case, we choose $I_{\text {asymp }}$ so that all panels reach peak pressure before failure as the impulse asymptote is reached.

The three wall-failure criteria were then used for the wall panels in simulations, while the roofs were kept as non-failing.

\subsection{Results with wall failure}

Figure 12a shows the maximum force and impulse reached on each roof panel with walls having the 'high impulse' failure criteria (see Table 1). Panel 30 has lower maximum force and lower maximum impulse than panels that did not fail, so we cannot draw a set of asymptotes that divide the failing from non-failing panels. This leads us to reject this failure criteria.

For the 'low impulse' wall-failure criteria (not shown for brevity), it is possible to draw asymptotes that divide the failing panels from the non-failing panels. However, the force asymptote value is tightly constrained.

Figure $12 \mathrm{~b}$ shows the maximum force and impulse reached on each roof panel using the 'mid impulse' criteria for wall failure. These criteria give us the best separation between the failing and non-failing panels, with a sizeable gap between the maximum force experienced by panels 30 and 29. These failure criteria seem to offer the best chance of setting up an appropriate failure model for the roof panels. 
Table 1: Pressure and impulse asymptotes for different wall-failure criteria.

\begin{tabular}{lll}
\hline Name & $p_{\text {asymp }}[\mathrm{kPa}]$ & $I_{\text {asymp }}[\mathrm{kPa} \cdot \mathrm{ms}]$ \\
\hline High Impulse & 600 & 240 \\
Low Impulse & 600 & 1 \\
Mid Impulse & 826.90 & 74.73 \\
\hline
\end{tabular}

Before applying the roof failure model, we consider the impact of failure on the overpressure time series at the pressure gauges: Q4's is shown in Fig. 13. The experimental overpressure is shown, as well as the simulated overpressure with no failure and with each set of the wall-failure criteria.

There is good agreement between the overpressure simulated with no failure and the experimental overpressure. The 'high impulse' failure criteria are also satisfactory: it is visually indistinguishable from the time series without failure until $100 \mathrm{~ms}$ and diverges significantly only after $140 \mathrm{~ms}$. The other failure criteria do not match the experimental results well. Decreasing the impulse asymptote decreases the peak pressure at this pressure gauge - this is typical of all pressure gauges examined. Examining this evidence leads us to reject the mid and low impulse criteria.

We see here two contradictory effects:

1. Wall panels must fail early enough for high pressures (forces) and impulses to be reached inside the buildings to produce the observed roof failures.

2. But later failure is needed to ensure sufficient energy is reflected from the failing panels so the overpressures and arrival times experienced downstream are maintained.

This is why the location of panel 30 in Fig. 12a and Fig. 12b differs significantly. With 'high impulse' failure criteria, the blast wave has already passed panel 13 when the panel breaks, so little of it enters building 3, and the force and impulse on building 3's roof panel 30 are low. 'Mid impulse' criteria allow panel 13 to fail quickly, so the blast wave travels inside of building 3, resulting in a larger force and impulse on panel 30.

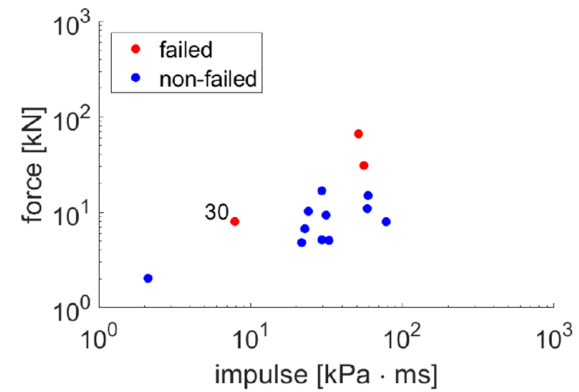

(a)

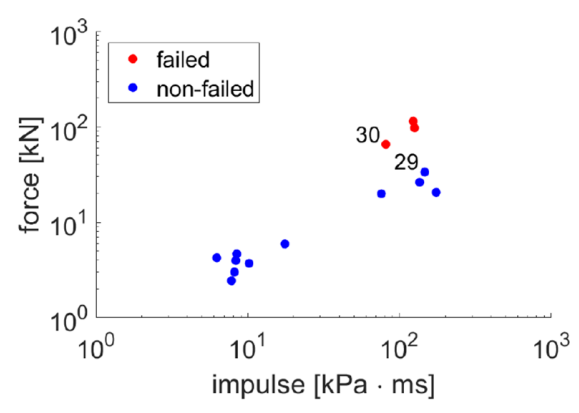

(b)

Figure 12: The maximum force and impulse reached on each failed (red) and non-failed (blue) roof panel during a $1.0 \mathrm{~cm}$ cell size simulation. (a) 'High impulse' wallfailure criteria; (b) 'mid impulse' wall-failure criteria. 


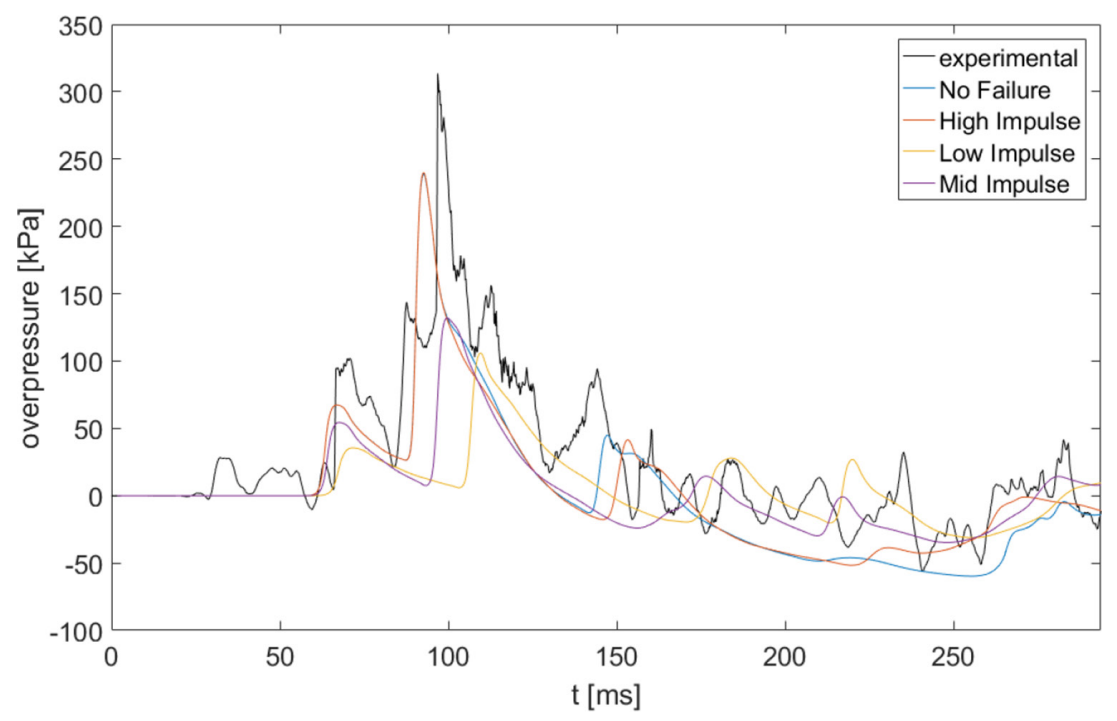

Figure 13: The simulated and experimental overpressure at Q4 using various different wallfailure criteria. The cell size used for these simulations was $1.0 \mathrm{~cm}$, which is equivalent to the $0.5 \mathrm{~m}$ resolution in the full-scale results presented in the previous sections.

Our current failure model cannot produce failure criteria that satisfy both of these factors: it is too simple to model this problem. For example, each panel could have a different P-I curve so that panel 30 fails because it is weaker than all of the other roof panels, not because its loading is higher. Also, we ignore the likely lag between reaching the threshold and failure occurring: physically the threshold pressure and impulse set the panel in motion such that it continues to reflect the shock wave until it later deflects sufficiently to fail.

\section{CONCLUSION}

We study simulated and experimental blast loading within a geometrically complex domain inspired by a city-center location. As in previous work [3], [4], elevated pressures at locations without line of sight to the original charge location confirm that the CFD-based approaches are superior to empirical, line-of-sight models in such cases.

Some buildings in the experimental model immediately adjacent to the blast suffered significant blast damage. These buildings either had their front and top faces blown off, exhibited buckling of those faces or were displaced from their initial positions. We expected this damage to produce differences between the experiment and simulation by first absorbing the energy of the blast and second altering the geometry through which the blast wave propagated.

Even with building failure, there is reasonable agreement between the simulated and experimental peak pressures for phase 1 , with the relative error between these two values around $20 \%$, and with plausible explanations behind values above this threshold. The phase 1 impulse shows worse agreement, with relative errors around $40 \%$. It appears the simulated data are applicable up until peak pressure is reached, but the effects of building damage become significant toward the end of phase 1. This appears reasonable, as failure might be expected at some time between the time of peak pressure and the end of phase 1, when the 
impulse reaches a local maximum. The agreement between the experimental results and the simulated loading is good, considering the amount of damage sustained by the buildings in the model.

While ProSAir's simple pressure-impulse curve failure model may be sufficient for glass windows, it could not model the experimentally observed failure. We believe this is largely due to two modeling errors:

1. The lack of an adequate P-I failure criteria for each plywood panel and structural member as there were multiple failure mechanisms: some members failed in bending and shear, and some fixings failed as screws were ripped out.

2. A P-I failure criteria resulting in immediate removal of failed panels is likely not appropriate as complete failure would be slightly delayed; this effect will be more pronounced for flexible materials, like plywood, than for brittle materials, like glass.

To overcome these modeling errors likely requires fully coupling the blast loading and structural response.

\section{ACKNOWLEDGMENTS}

The authors thank Pool Re, for funding this research and related publication costs, and Guy Carpenter, for supplying the original geometry that was simplified for this work. The authors also thank the technicians and range officers at COTEC, in particular, Daniel Flynn, Andrew Fenwick, and Richard Winder, for constructing and conducting the reported experiment.

\section{REFERENCES}

[1] Forth, S.A., Johnson, S., Burrows, S.J. \& Sheldon, R.P., Cranfield University Centre of Excellence in Counterterrorism. Enhancing CBRNE Safety \& Security: Proceedings of the SICC 2017 Conference, eds. A. Malizia \& M. D'Arienzo, Springer International Publishing: Cham, pp. 317-323, 2018. https://doi.org/10.1007/978-3-319-91791-7

[2] Folkman, C., Terrorism modeling and risk management. Presented at RAA Catastrophe Modeling Conference, Orlando, USA, 2014. Online. www.slideshare.net/RMS_News/ terrorism-modeling-risk-management-presented-at-theraas-cat-modeling-conference-2014. Accessed on: 5 Jan. 2018.

[3] Remennikov, A.M. \& Rose, T.A., Modelling blast loads on buildings in complex city geometries. Computers \& Structures, 83(27), pp. 2197-2205, 2005. https://doi. org/10.1016/j.compstruc.2005.04.003

[4] Rose, T. \& Smith, P., Influence of the principal geometrical parameters of straight city streets on positive and negative phase blast wave impulses. International Journal of Impact Engineering, 27(4), pp. 359-376, 2002. https://doi.org/10.1016/S0734743X(01)00060-4

[5] Forth, S., ProSAir computational blast loading tool website. Cranfield University, Shrivenham, UK. www.cranfield.ac.uk/facilities/cds-prosair-computational-blast-loading-tool. Accessed on: 5 Jan. 2018.

[6] Rose, T.A., An Approach to the evaluation of blast loads on finite and semi-infinite structures. PhD Thesis, Cranfield University, Cranfield Defence and Security, Shrivenham, UK, 2010. https://dspace.lib.cranfield.ac.uk/handle/1826/4262

[7] Forth, S., ProSAir 2018.4 User Guide. Cranfield Defence and Security, Cranfield University, Shrivenham, UK, 2018. 
[8] Smith, P., Rose, T. \& Brittle, M., Analysis of a generic cityscape using an adaptive mesh CFD code. Proceedings of the 12th International Symposium on Interaction of the Effect of Munitions with Structures, ISIEMS: New Orleans, LA, USA, 2005.

[9] Brittle, M., Blast propagation in a geometrically complex environment. Master's thesis, Cranfield University, Shrivenham, UK, 2004.

[10] S.J. Burrows, Forth, S. \& Sheldon, R., Data for "Partial Validation of CFD Blast Simulation in a Cityscape Environment Featuring Structural Failure". Cranfield Online Research Data (CORD), 2019. http://dx.doi.org/10.17862/cranfield.rd.8175026

[11] LeVeque, R.J., Finite Volume Methods for Hyperbolic Problems. Cambridge Texts in Applied Mathematics, Cambridge University Press, 2002.

[12] UN - Safeguard, Kingery Bulmash Blast Parameter Calculator. United Nations Office for Disarmament Affairs, New York. Online. www.un.org/disarmament/un-saferguard/ kingery-bulmash/. Accessed on: 13 Sep. 2017.

[13] Smith, D. \& Cormie, D., Design of glazing. Blast Effects on Buildings, eds. G. Mays, P.D. Smith \& D. Cormie, Thomas Telford Ltd, pp. 177-215, 2nd edition, 2009. https:// doi.org/10.1680/beob2e.35218.0009 